\title{
Evaluation of self-educational training methods to learn laparoscopic skills - a randomized controlled trial
}

\author{
Steffen Axt ${ }^{1}$, Pirmin Storz ${ }^{1}$, Carolin Ehrenberg ${ }^{1}$, Claudius Falch', Marc Immenroth², Andreas Kirschniak ${ }^{1 *}$ \\ and Sven Muller ${ }^{1}$
}

\begin{abstract}
Background: Evaluation of two different self-educational methods (video assisted learning versus video assisted learning plus a nodal point operation primer) on learning laparoscopic suturing and intracorporal knotting.

Methods: Randomized controlled trial at the laparoscopic surgical training center, University of Tubingen with 45 surgical novices first year medical students being pretested for dexterity. After self-educational training for 90 min with either method (Group A: video assisted learning, Group B: video assisted learning plus a nodal point operation primer) participants had to perform five laparoscopic intracorporal knots. Assessed were number of knots completed (maximum of five knots counted, knot integrity, technical proficiency and knotting time per knot. Primary outcome measure is a composed knot score combining knot integrity, technical proficiency and knotting time.

Results: Group B ( $n=23)$ achieved a significantly higher composed knot score than Group A $(n=22)(53.3 \pm 8.4$ versus $46.5 \pm 13.6$ points respectively, $p=0.016$ ). Median knotting time per completed knot was significantly different between Group B and Group A (308 s [100-1221] versus 394 s [138-1397] respectively, $p=0.001$ ). Concerning number of completed knots there was a trend towards more knots achieved in Group B (4.2 \pm 1.2 versus $3.55 \pm 1.4$ respectively, $p=0.075$ ).

Conclusions: The use of a nodal point operation primer highlighting essential key steps of a procedure augment the success of learning laparoscopic skills as suturing and intracorporal knotting. (UIN researchregistry3866, March 22, 2018).
\end{abstract}

Keywords: Laparoscopic suturing and intracorporal knotting, Operation primer, Self-educational, Video-assisted learning

\section{Background}

Learning laparoscopic surgical skills is a major challenge for interns and of paramount importance in any educational surgical residency program. Especially in the light of limited training opportunities in the operation room due to restricted working hours and operation costs at its limit, optimized resident teaching strategies are warranted [1]. Simulation based training methods have been published that support training success and transferability into the operation room [2]. However the instructional

\footnotetext{
* Correspondence: akirschniak@me.com; andreas.kirschniak@med.unituebingen.de

${ }^{1}$ Working Group for Surgical Technique and Training, Clinic for Visceral, General and Transplant Surgery, Tuebingen University Hospital, 72072 Tübingen, Germany

Full list of author information is available at the end of the article
}

implementation strategies of simulation based resident training are less well investigated. Self-educational learning by video tutoring and following hands on training by the resident himself is one possible option [3]. An additional available teaching approach is "step-by-step"instructions based on the philosophy of the "mental training", repeatingly internalizing the essential key points of a procedure. Though, the actual mental training requires in the optimum case a "one-to-one" personal coaching and its practicability is therefor limited in everyday use.

The nodal point concept breaking down a procedure in a limited number of essential key steps significantly improves the learning and internalization of laparoscopic skills. This concept highlights crucial steps of a procedure and helps to repeatedly imagine the procedure [4].

(C) The Author(s). 2018 Open Access This article is distributed under the terms of the Creative Commons Attribution 4.0 International License (http://creativecommons.org/licenses/by/4.0/), which permits unrestricted use, distribution, and 
The aim of this randomized controlled trial was to assess the value of an additional "nodal point operation primer" without the actual mental training in the process of video assisted self-educational learning of laparoscopic suturing and intracorporal knotting.

\section{Methods}

\section{Setting and participants}

This study was conducted by the Surgical Training Center at the University Tubingen. Because of the educational nature of the study, evaluation of the study protocol by the local ethics committee was not necessary. The participants were all first year medical students without pre-existing experiences in surgical techniques and especially no previous laparoscopic skills.

\section{Baseline assessment and pretests}

In preparation all study participants received an instructional video to teach elementary basics of laparoscopy and laparoscopic suturing and intracorporal knotting. Afterwards, participants had to reply a standardized questionnaire about basic laparoscopy before study inclusion. Exclusion from the study was done if less than $60 \%$ of questions were correctly answered. After study inclusion two preliminary simulation based laparoscopic exercises and a laparoscopic intracorporal knot were performed in a black box trainer to assess baseline manual dexterity and the ability of three-dimensional orientation. For the first preliminary simulation based laparoscopic exercise participants had to touch 20 contact points of a 3-D model, in which every contact point had a diameter of $3 \mathrm{~mm}$ surrounded by a ring which could be illuminated by LEDs. Contacting the surrounding of the point was electronically detected as an error. For the second preliminary simulation based laparoscopic exercise the participants had to pass a needle horizontally through five openings in a wall with two grasping instruments. Touching the wall with the needle or the instruments was electronically detected as an error. In both exercises, the number of errors and the necessary performing time in seconds were determined. Finally the participants had to perform a pretest laparoscopic intracorporal knot in a maximum time span of seven minutes according to the below mentioned criteria after watching an uncommented video demonstrating laparoscopic suturing and intracorporal knotting.

\section{Randomization}

After study inclusion and successful pretesting participants were randomly allocated to either group A (video assisted learning) or group B (video assisted learning plus operation primer) in a 1:1 ratio.

\section{Study performance}

Participants of both groups received a standardized introduction to the laparoscopic workstation for $10 \mathrm{~min}$. From now on Group A and B were separated for the learning phase and final testing.

\section{Group a - Video assisted learning}

Participants of Group A watched a commented video of the performance of laparoscopic suturing and intracorporal knotting. Afterwards the video was shown during a training period of $90 \mathrm{~min}$ in a continuous loop so that a presentation of the performance was available for the participants all the time.

\section{Group B - video assisted learning plus a key point operation primer}

Additionally to the video, the participants of Group B received an operation primer booklet highlighting the 10 key points of laparoscopic suturing and intracorporal knotting in text form and with example pictures (see Additional File 1). The concept of an operation primer is based on highlighting crucial steps of a procedure by key points and helps to repeatedly imagine the procedure. According to Immenroth et al. the definition of a key point is that it represents necessary structural motor components that must be performed in sequence and are marked by a reduction in the degrees of freedom of action (e.g correct alignement of the needle with the needle holder is a mandatory steps for a correct puncture of the wound edge in the following step) [4].

The correct use of the operation primer was shown in a standardized video presentation. Subsequently the participants had $90 \mathrm{~min}$ to train laparoscopic suturing and knots according to the video and the operation primer.

Finally after a 20 min break, each participant was instructed to perform five laparoscopic intracorporal knots in a maximum time span of 35 min without further help. The performance was video-taped for further evaluation.

\section{Outcome measure}

The analysis of the videotapes and the knot specimen was performed independently by two blinded investigators (both board certified general surgeons). Assessed were the number of knots completed (maximum of five knots counted), knot integrity, technical proficiency and knotting time per knot.

The primary end point was a composed knot score combining knot integrity, the technical proficiency and the knotting time. Knot integrity was defined by a sufficient tightened and squared knot defined as, complete adaption of the wound margins (adaption of the sponge edged [knot not too loose]), correct distance of the needle injections, no tears in the sponge (knot too tight) as 
well as sufficient length of the rest of the suture $(5 \mathrm{~mm})$. A maximum of 6 points (one for each fulfilled evaluation criterion) could be reached for knot integrity.

Technical proficiency was defined as correctly performing the 10 key steps shown in the video and highlighted in the operation primer in the correct order. For reaching a step one could get two points. In addition, an extra-point was awarded for the right performance of the essential part of a step of the knotting technique. Thus, a maximum score of 30 points could be reached for technical proficiency.

Knotting time per knot was defined as time needed for the performance of one complete knot (knotting time) and was recorded in seconds. The knotting time was then transferred by means of a conversion table in a point value. The maximum achievable points given were 30 for a time needed of $<209 \mathrm{~s}$ for one knot. For each additional $35 \mathrm{~s}$ time needed a point was deducted.

As a maximum result a score of 66 points was possible. Only completely performed knots were evaluated for knot integrity, technical proficiency, knotting time and number of correctly performed knots.

The mentioned composed knot score used at the Surgical Training Center at the University Tubingen to compare the before/after results of laparoscopic knotting in the residency training program is similar to the knot score proposed by Aggarwal et al. [5].

\section{Materials}

As suture material a 3-0 polyfilament suture with a $26 \mathrm{~mm}$ in diameter SH-needle (Ethicon Endo Surgery; Johnson and Johnson, New Brunswick; New Jersey, USA) was used. The length of the suture was standardized to $120 \mathrm{~mm}$. The knots were performed on sponges with marks of $5 \mathrm{~cm}$ width and $1 \mathrm{~cm}$ height, which defined the area in which the knots had to be performed and in which a $8 \mathrm{~cm}$ long incision was placed in the center. The laparoscopic tower was equipped by Karl Storz endoscopes (Karl Storz Endoscopes GmbH and Co. KG, Tuttlingen, Germany). The equipment consisted of high density cameras (Karl Storz H3 Z Full-HD, Karl Storz SCB Image 1 HD Hub 222,010 20), 30-optics (Karl Storz Hopkins ${ }^{\circ}$-II $30^{\circ}$-optics) and cold light sources (Karl Storz SCB xenon 300 20,133,120). The recordings were done by an integrated reception system (AIDA, Karl Storz Endoscopes GmbH and Co. KG, Tuttlingen, Germany). During the performance of the study knots, the optics were fixed in an angle of $45^{\circ}$ by a Karl Storz Martins arm (Karl Storz Endoscopes $\mathrm{GmbH}$ and Co. KG, Tuttlingen, Germany). The used laparoscopic instruments were a needle holder (Karl Storz, needle holder to Koh Marco; straight grip), a laparoscopic atraumatic grasping forceps (Karl Storz; click-Line Dissecting and Grasping Forceps according to
Kelly, pistol grip) and a laparoscopic scissors (Karl Storz, click shear line; pistol grip).

\section{Statistical evaluation}

Sample size calculation was done assuming a difference for the primary end point between group A and group B of greater than $20 \%$ to be relevant. This difference could be detected with a two-sided significance level $\alpha=0.05$ and a power of $1-\beta=0.9$, with a group size of 22 participants randomized to each group. The statistical evaluation was performed with SPSS 21 (SPSS Inc., Chicago, IL, USA). First the arithmetic means and their standard deviations or medians and their interquartile ranges were determined. In the analytical statistics first the determination of the normal distribution was done. Subsequently, the test of homogeneity of variance followed. Significance was tested by the student t-test for independent samples or the Mann-Whitney-U-test for independent samples. The study was registered at www. researchregistry.com (UIN researchregistry3866).

\section{Results}

Flow of participants through the study is displayed in Fig. 1. Participant characteristics and pretest results were well distributed and are shown in Table 1. None of the participants achieved to perform a complete pretest knot. Outcome measures are summarized in Table 2. For the primary outcome measure composed knot score group B $(n=23)$ achieved significantly more total points than group A $(n=22)(53.3 \pm 8.4$ versus $46.5 \pm 13.6$ points respectively, $p=0.016)$. Especially median knotting time per completed knot was significant different between Group B and Group A (308 s [100-1221] versus 394 s [138-1397] respectively, $p=0.001$ ). Concerning number of completed knots there was a trend towards more knots achieved in Group B $(4.2 \pm 1.2$ versus $3.55 \pm 1.4$ respectively, $p=0.075)$. Concerning the inter-rater reliability of the two blinded reviewers for the outcome measures, there was discordance in only one case judging tears in the sponge.

\section{Discussion}

The present trial shows that successful self-educational video assisted learning of laparoscopic skills with an additional operation primer highlighting the key points of the procedure can augment the trainees perfomance. Several studies have shown that simulation based laparoscopic training significantly improves the performance of residents and surgical beginners in the operation theatre $[6,7]$. A main reason for the increasing number of available additional training tools is the financial pressure of hospitals that cannot offer surgical training any more exclusively in the operating room $[1,8]$. However there are numerous training approaches for the acquisition of 


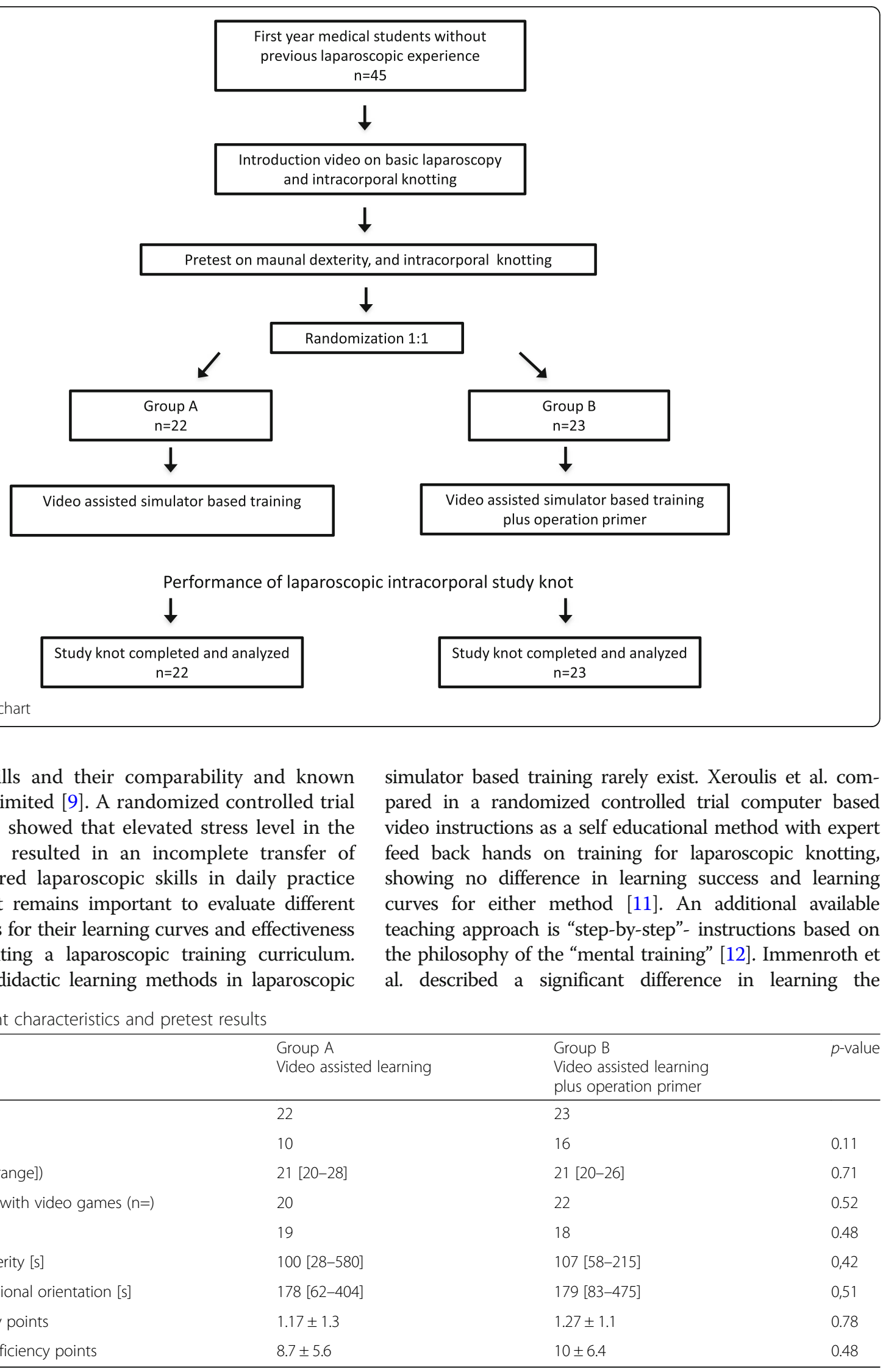

laparoscopic skills and their comparability and known effectiveness is limited [9]. A randomized controlled trial by Prabhu et al. showed that elevated stress level in the operating room resulted in an incomplete transfer of simulator acquired laparoscopic skills in daily practice [10]. Therefor it remains important to evaluate different training methods for their learning curves and effectiveness when implementing a laparoscopic training curriculum. Studies on autodidactic learning methods in laparoscopic simulator based training rarely exist. Xeroulis et al. compared in a randomized controlled trial computer based video instructions as a self educational method with expert feed back hands on training for laparoscopic knotting, showing no difference in learning success and learning curves for either method [11]. An additional available teaching approach is "step-by-step"- instructions based on the philosophy of the "mental training" [12]. Immenroth et

Table 1 Participant characteristics and pretest results

\begin{tabular}{llll}
\hline & Group A & Group B & $\begin{array}{l}\text { Video assisted learning } \\
\text { plus operation primer }\end{array}$ \\
\hline $\mathrm{N}=$ & & 23 & 16 \\
Female Gender & 22 & $21[20-26]$ & 0.11 \\
Median age (years, [range]) & 10 & 22 & 0.71 \\
Previous experience with video games (n=) & $21[20-28]$ & 18 & 0.52 \\
Right handed (n=) & 20 & $107[58-215]$ & 0.48 \\
Pretest manual dexterity [s] & 19 & $179[83-475]$ & 0,42 \\
Pretest three-dimensional orientation [s] & $100[28-580]$ & $1.27 \pm 1.1$ & 0,51 \\
Pretest knot integrity points & $178[62-404]$ & $10 \pm 6.4$ & 0.78 \\
Pretest technical proficiency points & $1.17 \pm 1.3$ & 0.48 \\
\hline
\end{tabular}


Table 2 Participant outcome measures

\begin{tabular}{llll}
\hline & $\begin{array}{l}\text { Group A } \\
\text { Video assisted learning }\end{array}$ & $\begin{array}{l}\text { Group B } \\
\text { Video assisted learning } \\
\text { + operation primer }\end{array}$ \\
\hline $\mathrm{n}$ & 22 & 23 \\
Total composed knot points & $46.5 \pm 13.6$ & $53.3 \pm 8.4$ & $3.4 \pm 0.6$ \\
Knot integrity points & $3.18 \pm 1.2$ & $24.3 \pm 2.9$ & 0.016 \\
Technical proficiency points & $22.7 \pm 5.6$ & $25.5 \pm 6.5$ & 30.76 \\
Knotting time points & $20.6 \pm 8.6$ & $308[100-1221]$ & 0.22 \\
Mean Knotting time per knot (sec) & $394[138-1397]$ & $4.2 \pm 1.2$ & 0.009 \\
Number of correctly performed knots & $3.55 \pm 1.4$ & & 0.001 \\
\hline
\end{tabular}

laparoscopic cholecystectomy between practical and mental training [4]. Though, mental training requires in the optimum case a "one-to-one" personal coaching and its practicability is therefor limited in everyday use. Based on this principal of "step-by-step" - instructional mental training the concept of a nodal point operation primer divides and highlights a procedure in a limited number of essential key steps without the time and human resource consuming relaxation and repetition exercises. The aim of such a nodal point operation primer is a deeper and more structured internalization of the procedure by breaking it down to its essential 10 key points and therefore a higher and more sustainable chance of reproduction during a stressful situation [13]. Our results underline that for medical novices the acquisition of laparoscopic skills such as intracorporal suturing and knot tying is improved using an additional operation primer. Especially the time needed to perform a laparoscopic knot was significantly shorter and there was a trend towards more correctly performed knots in a predefined time period in the operation primer group.

A potential bias of this study is that during the practice period no data about the amount of time spent on the task of laparoscopic knotting and on the actual usage of the video and/or the operation primer are available. However this study was primarily designed to evaluate two selfeducational methods of learning laparoscopic knotting accepting that the amount of resource used by each single participant might be different among the two groups.

Simulation based training irrespective of the method itself has been shown to have a positive effect on all aspects of laparoscopic learning and its transferability of the acquired skills to the operation room [14]. Akl et al. reported that by just showing an instructional and structured video to the participants before training a significant reduction of laparoscopic knotting time was achieved [15]. Further, limited evidence exists on the influence of different teaching methods used in simulator based laparoscopic training on long term skill retention. While repetitive hands-on training is shown to be more proficient, the amount of instructor feedback seems to have no impact long term laparoscopic skill retention $[14,16]$. The effect of a nodal point operation timer on long-term skill retention has to be issue of further evaluation.

\section{Conclusion}

Altogether, the use of a nodal point operation primer highlighting essential key steps of a procedure augments the success of performing laparoscopic skills as suturing and intracorporal knotting. It should therefor be implemented in self-educational simulation based laparoscopic teaching programs as it is a valuable tool in taking up a procedure.

\section{Additional file}

Additional file 1: Institutional education booklet on laparoscopic suturing and knotting. An institutional education booklet explaining ten key steps of laparoscopic suturing and knotting. We have permission for publication of this institutional booklet from the University hospital Tuebingen, Germany. (PDF 1595 kb)

\section{Acknowledgements}

The authors wish to extend their thanks to all participants in the study and the support staff of the Surgical Training Center Tuebingen for their invaluable assistance.

\section{Funding}

There was no funding for the study.

\section{Availability of data and materials}

All the data on which the conclusions of this manuscript rely are available without restrictions by contacting the corresponding author.

\section{Authors' contributions}

SA contributed by designing and planning the study, collecting data, performing the statistical analyses and interpretation, writing and revising the manuscript, and final approval of the submitted manuscript. PS, CE, CF \& $\mathrm{Ml}$ contributed by planning the study, interpretation of statistical analysis and final approval of the submitted manuscript. SM \& AK contributed by designing, facilitating and planning the study, collecting data, performing the statistical analyses and interpretation, writing and revising the manuscript, and final approval of the submitted manuscript. All authors read and approved the final manuscript.

Ethics approval and consent to participate

All participants verbally consented to participating in the study, which was approved by the local Ethics Committee University Tuebingen. No additional approval from the local Ethics Committee University Tuebingen was necessary. 


\section{Competing interests}

The authors declare that they have no competing interests.

\section{Publisher's Note}

Springer Nature remains neutral with regard to jurisdictional claims in published maps and institutional affiliations.

\section{Author details}

'Working Group for Surgical Technique and Training, Clinic for Visceral, General and Transplant Surgery, Tuebingen University Hospital, 72072

Tübingen, Germany. ${ }^{2}$ Ethicon, Endo-Surgery Europe, Norderstedt, Germany.

Received: 27 July 2016 Accepted: 18 April 2018

Published online: 02 May 2018

\section{References}

1. Babineau TJ, Becker J, Gibbons G, Sentovich S, Hess D, Robertson S, et al. The "cost" of operative training for surgical residents. Arch Surg. 2004;139(4): 366-9; discussion 9-70.

2. Stefanidis D, Scerbo MW, Montero PN, Acker CE, Smith WD. Simulator training to automaticity leads to improved skill transfer compared with traditional proficiency-based training: a randomized controlled trial. Ann Surg. 2012;255(1):30-7

3. Singh P, Aggarwal R, Tahir M, Pucher PH, Darzi A. A randomized controlled study to evaluate the role of video-based coaching in training laparoscopic skills. Ann Surg. 2015:261(5):862-9.

4. Immenroth M, Burger T, Brenner J, Nagelschmidt M, Eberspacher $H_{\text {, Troidl }}$ H. Mental training in surgical education: a randomized controlled trial. Ann Surg. 2007;245(3):385-91

5. Aggarwal R, Hance J, Undre S, Ratnasothy J, Moorthy K, Chang A, et al. Training junior operative residents in laparoscopic suturing skills is feasible and efficacious. Surgery. 2006;139(6):729-34.

6. McGaghie WC, Issenberg SB, Cohen ER, Barsuk JH, Wayne DB. Does simulation-based medical education with deliberate practice yield better results than traditional clinical education? A meta-analytic comparative review of the evidence. Acad Med. 2011;86(6):706-11.

7. Sturm LP, Windsor JA, Cosman PH, Cregan P, Hewett PJ, Maddern GJA Systematic review of skills transfer after surgical simulation training. Ann Surg. 2008;248(2):166-79.

8. Scott DJ, Bergen PC, Rege RV, Laycock R, Tesfay ST, Valentine RJ, et al. Laparoscopic training on bench models: better and more cost effective than operating room experience. J Am Coll Surg. 2000;191(3):272-83.

9. Willis RE, Richa J, Oppeltz R, Nguyen P, Wagner K, Van Sickle KR, et al. Comparing three pedagogical approaches to psychomotor skills acquisition. Am J Surg. 2012;203(1):8-13.

10. Prabhu A, Smith W, Yurko Y, Acker C, Stefanidis D. Increased stress levels may explain the incomplete transfer of simulator-acquired skill to the operating room. Surgery. 2010;147(5):640-5.

11. Xeroulis GJ, Park J, Moulton CA, Reznick RK, Leblanc V, Dubrowski A. Teaching suturing and knot-tying skills to medical students: a randomized controlled study comparing computer-based video instruction and (concurrent and summary) expert feedback. Surgery. 2007;141(4):442-9.

12. Hall JC. Imagery practice and the development of surgical skills. Am J Surg 2002;184(5):465-70.

13. Niwa UC, Axt S, Falch C, Muller S, Kreuzer JA, Nedela P, et al. Laparoscopic cholecystectomy as standardised teaching operation to treat symptomatic cholecystolithiasis. Zentralbl Chir. 2013;138(2):141-2.

14. Zendejas B, Brydges R, Hamstra SJ, Cook DA. State of the evidence on simulation-based training for laparoscopic surgery: a systematic review. Ann Surg. 2013;257(4):586-93

15. Akl MN, Giles DL, Long JB, Magrina JF, Kho RM. The efficacy of viewing an educational video as a method for the acquisition of basic laparoscopic suturing skills. J Minim Invasive Gynecol. 2008;15(4):410-3.

16. Stefanidis D, Korndorffer JR Jr, Markley S, Sierra R, Scott DJ. Proficiency maintenance: impact of ongoing simulator training on laparoscopic skil retention. J Am Coll Surg. 2006;201(4):599-603.

\section{Ready to submit your research? Choose BMC and benefit from:}

- fast, convenient online submission

- thorough peer review by experienced researchers in your field

- rapid publication on acceptance

- support for research data, including large and complex data types

- gold Open Access which fosters wider collaboration and increased citations

- maximum visibility for your research: over $100 \mathrm{M}$ website views per year

At BMC, research is always in progress.

Learn more biomedcentral.com/submissions 